\title{
Haptic Perception of Virtual Spring Stiffness Using ExoTen-Glove
}

\author{
$1^{\text {st }}$ Mohssen Hosseini \\ Robotics Research Group* \\ Department of Mechanical Engineering \\ KU LEUVEN, Belgium. \\ mohssen.hosseini@kuleuven.be \\ $4^{\text {th }}$ Joris De Schutter \\ Robotics Research Group \\ Department of Mechanical Engineering \\ KU LEUVEN, Belgium. \\ joris.deschutter@kuleuven.be
}

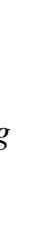

\author{
$2^{\text {rd }}$ Ali Sengül \\ Helbling Technik AG, Switzerland. \\ ali.senguel@helbling.ch
}

\author{
$5^{\text {th }}$ Herman Bruyninckx \\ Robotics Research Group \\ Department of Mechanical Engineering \\ KU LEUVEN, Belgium / TU/e, The Netherlands. \\ herman.bruyninckx@kuleuven.be
}

\begin{abstract}
This paper presents a study of haptic perception of virtual stiffness and the influence of visual feedback in virtual reality. In this study, a novel and lightweight haptic glove (ExoTen-Glove) based on Twisted String Actuation (TSA) system is presented and evaluated. This system has two independent TSA modules with integrated force sensors and small-size DC motors. ExoTen-Glove provides force feedback to the users during the execution of grasping virtual objects. The overall design, the controller and the preliminary experimental evaluation of the ExoTen-Glove have been shown in this paper. Different experiments have been performed in virtual reality environment using HTC VIVE headset with 2 degrees of freedom grasping tasks, squeezing a pair of virtual springs with the participant's thumb and index fingers. The aim of this study is to illustrate the benefit of using ExoTen-Glove to distinguish stiffness of a pair of virtual springs and the role of the visual feedback. The results show that the users use not only haptic cues but also use visual cues in detecting spring stiffness difference.
\end{abstract}

Index Terms-Force feedback, Twisted String Actuation System, Wearable, Virtual Reality, Stiffness discrimination, Tendon Transmission System, Human Computer Interaction, Human haptic perception.

\section{INTRODUCTION}

Virtual Reality (VR) technology has been explored since late 1950's and has been exploited in a wide range of applications. VR can simulate the real world dynamically and response in real time to the users. In virtual reality interaction, haptic feedback is a crucial sensory modality. Haptic devices are electromechanical systems that make it possible to touch, manipulate and interact with virtual objects. They provide a physical contact with the user to measure the position of the user and enable them to interact with virtual object with electrical connection by simulating the interaction forces back to the users. Haptics are used in various applications, such as entertainment (gaming industry), medical (surgical training), automotive, academic

\footnotetext{
* Robotics Research Group is a university core lab of Flanders Make.
}

978-1-5386-5024-0/18/\$31.00 @2018 IEEE research, scientific visualizations. Different kind of feedback can be provided, such as force [1]-[3], tactile [4]-[6] or pressure feedback [7], [8].

Researchers have been developed various haptic interfaces for human hands over the past few years, for telemanipulation [9], [10], for medical training [11], [12] and for interaction with nano and micro scale phenomenon [13], [14]. The most used haptic interface is the PHANToM [15] that provides a force feedback on the finger, allowing the users to feel the stiffness and textures of virtual objects.

Wearable haptic devices with force feedback allow the user to grasp, feel, and even manipulate virtual objects in a more natural way. A commercial grounded force feedback system, CyberGrasp [16], has been presented that uses cable-driven exoskeleton structure on the back of the hand and can be combined with a separately available CyberGlove data glove, providing joint angle information of the hand and fingers with a full wrist closure. The Rutgers Master II-ND glove [17] is a haptic interface, developed at Rutgers University, designed for dexterous interactions with virtual environments using pneumatic actuators. Choi et al. implemented Wolverine, a lightweight, low-cost and wearable haptic device to simulate the grasping rigid objects in virtual environment that is able to provide about $100 \mathrm{~N}$ force directly between the thumb and three fingers [18]. DEXMO [19], is a mechanical exoskeleton system that has been implemented for motion capturing and force feedback for virtual reality.

In this paper we exploited a haptic glove (ExoTen-Glove) based on TSA system to study the perception of stiffness and the effect of visual feedback while squeezing virtual springs. The TSA system [20], [21] implements a lightweight, lowcost and powerful tendon-based driving system that uses small DC motors characterized by high-speed, low-torque and limited inertia. It has been used in various application such as DEXMART hand [22], 1-DOF elbow exoskeleton [23] and a 6-DOF cable-driven haptic interface [24]. The ExoTen-Glove is able to provide the users force feedback 


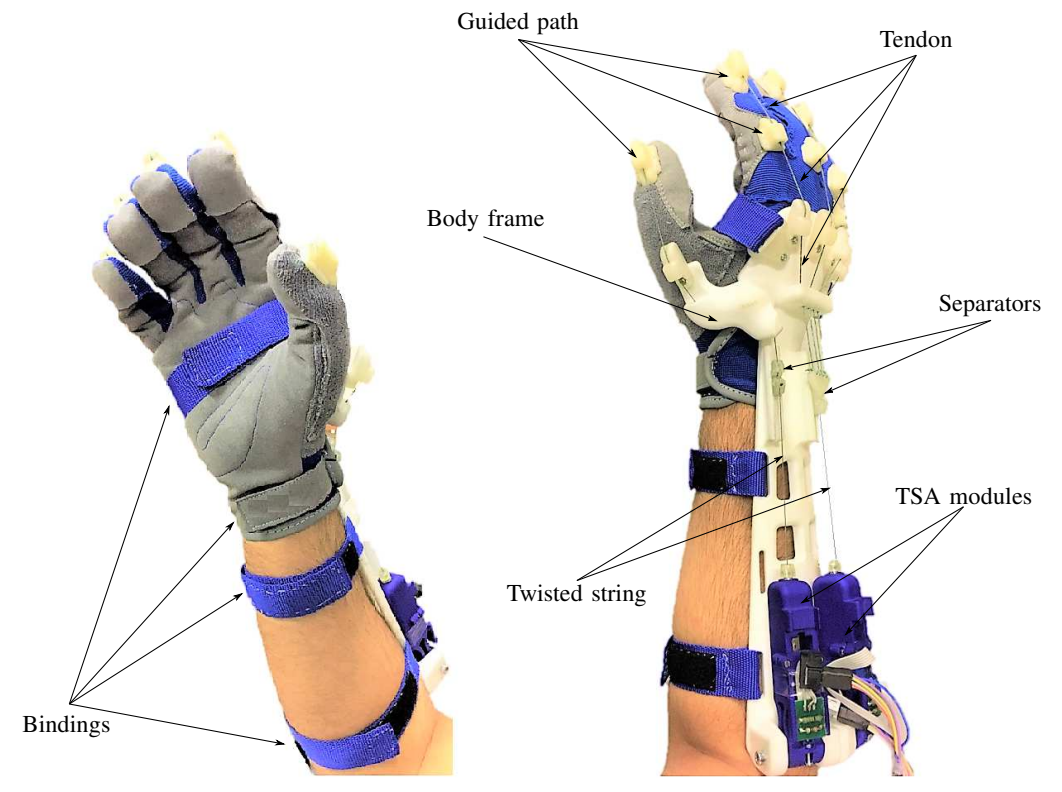

Fig. 1. Overview of the TSA-based haptic glove.

during the execution of grasping tasks in a virtual reality environment and it is based on a simple design without any bulky mechanism, like gears, relying on the skeleton structure of the human hand.

Recent developments in virtual reality made it possible to have an increased immersive virtual interaction by providing multi-sensory interactions such as visual, auditory and tactile. As most human interfaces use information of visual and auditory sensation while adding force/tactile sensation, the users can get more realistic feelings and perform complex tasks during interaction with the virtual environment. Hence, in this study, the effect of visual feedback in virtual stiffness perception using ExoTen-Glove is evaluated. We expect that the users would use both haptic and visual cues in stiffness perception and they would have higher accuracy when the visual and haptic cues are coinciding.

The paper is structured as follows. Section II describes the design of the overall system. In Section III experimental evaluation of the device is reported and finally, Section IV provides the conclusions and discusses future research activities.

\section{Description Of The System}

\section{A. ExoTen-Glove}

Figure 1 presents a lightweight and simple haptic glove (ExoTen-Glove) based on Twisted String Actuation (TSA) system. The TSA system is a low-cost, lightweight and compact linear transmission system that is using a small-size DC motor characterized by high speed and low torque. The proposed ExoTen-Glove has a very simple design without any external bulky mechanism to drive the fingers by tendons with a full range of motion, using skeleton structure of human fingers. The overall system is composed by (i) a rigid supporting frame with anatomical shape of forearm, made of Polyamide (PA 12) manufactured by Laser Sintering (SLS); (ii) a commercial soft gloves, to be worn by user and mount the tendon guidelines; (iii) two independent TSA modules [25], [26], to drive a group of fingers from index to pinky finger and the thumb separately for grasping tasks. The actuator consists of a frame (94 $\mathrm{mm}$ length, $24 \mathrm{~mm}$ height, $19 \mathrm{~mm}$ width) made of Plyamide (PA 12) manufactured by SLS (Selective Laser Sintering), a DC motor (maxon DCX) with encoder (maxon ENX), an integrated force sensor [27], [28] based on optoelectronic components (OMRON EE-SX1108). The total weight of the actuator is $40 \mathrm{~g}$; (iv) tendons (Dyneema with the thickness of $0.26 \mathrm{~mm}$ ), to connect the fingers to the actuators; (v) the bindings, for tightening; (vi) the curved shape guided path structure, made of semi-soft material 3D printed (EFLEX) that are attached on the glove itself. The shape has been chosen to allow the tendons follow the finger movement with its anatomical shape and prevent the shear forces to the attachment points; (vii) a linear guide, to prevent the twisting of the strings itself and with a separator to guide the tendons trough each finger.

The ExoTen-Glove is able to provide $80 \mathrm{~N}$ force from each actuator which is sufficient according to the human finger capability and its overall weight is $360 \mathrm{~g}$.

\section{B. Electronics and Controller}

Figure 2 represents the overall architecture of the ExoTenGlove. A STM32F4 32-bit microcontroller performs the signal processing and control computation. The force controller is realized using a cascaded PID control scheme with $1 \mathrm{kHz}$ force control loop and $5 \mathrm{kHz}$ velocity control loop. In order 


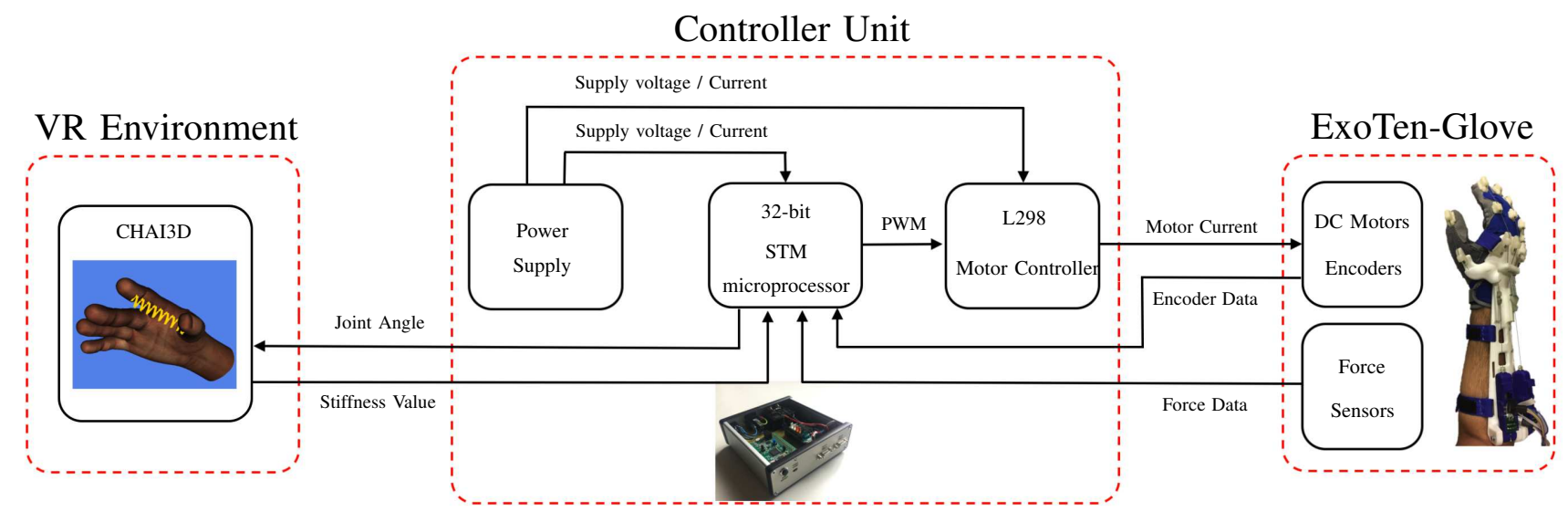

Fig. 2. Overall architecture of the ExoTen-Glove.

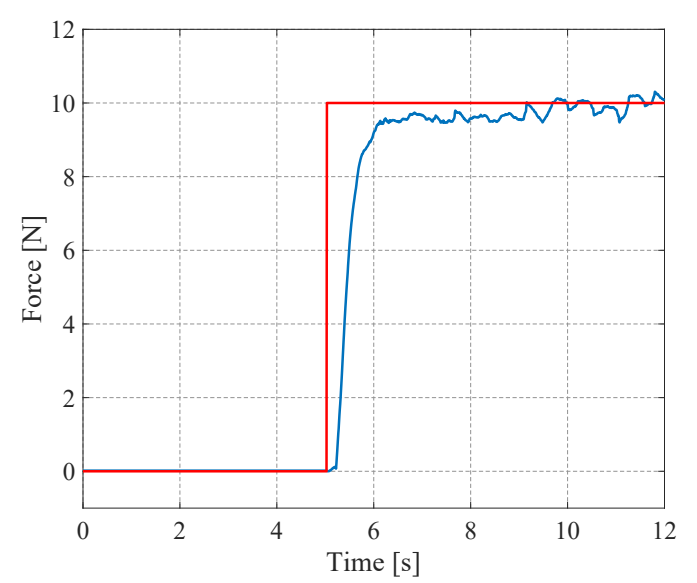

Fig. 3. The step response of the TSA force controller. The control bandwidth is approximately $2 \mathrm{rad} / \mathrm{s}$.

to obtain a satisfactory response, we determine that the time constant given a step reference signal should be at least 0.5 second. Based on this criteria, we then tune the $K_{p}, K_{i}$ and $K_{d}$ parameters using heuristic. Figure 3 depicts the force control response with respect to a step signal. As evident from the plot, the measured force tracks the desired value with satisfactory rise time and no visible overshoot.

\section{EXPERIMENTAL EVALUATION}

\section{A. Subjects}

8 healthy male subjects ( 7 right-handed and 1 left-handed), aged between 26 and 36 years old (average age of 31), participated to the experiment. There was no vision, touch disorder and history of neurological or psychiatric condition of all subjects. Each experiment took around 40 minutes for each participant and the general idea of the research was explained to the participants.

\section{B. Material}

ExoTen-Glove, a TSA-based haptic glove, is used for the experiment. It tracks the finger movements and can render higher forces during grasping without instability. The system is lightweight and provides realistic and transparent interaction in virtual reality. During the experiment, the participants were seated next to the setup table and wore the ExoTenGlove in their right hands. Participants were able to see their finger movements and interactions with virtual objects at HTC VIVE headset. The experiments were performed using a desktop PC, Intel(R) Xeon(R) $2.40 \mathrm{GHz} 2.39 \mathrm{GHz}$ CPU (2 processors), 24 GB of RAM, with NVIDIA GeForce GTX 1060 3GB Graphic card.

\section{Apparatus}

Virtual hand and springs were developed in CHAI3D that is a development environment for the modeling and simulating the haptics. Different commercial haptics devices are supported by CHAI3D. In order to make CHAI3D to support ExoTen-Glove which is a custom-made device, we extended it by adding the drivers of the ExoTen-Glove.

\section{Design of Experiments}

$2 \times 2$ factorial design was in the experiment. These factors were the spring stiffness simulated at the ExoTen-Glove(low stiffness vs. high stiffness) and congruency of the appearance of the virtual springs with respect to spring value felt at ExoTen-Glove (congruent vs. incongruent).

Participant wore the ExoTen-Glove and HTC VIVE headset during the experiment. They squeezed two virtual springs (with the stiffness of $2 \mathrm{~N} / \mathrm{mm}$ and $6 \mathrm{~N} / \mathrm{mm}$ ) with their thumb and index finger. Before starting the experiment, they were asked to become familiar with the stiffness value and the appearance of the virtual springs and memorize it while trying out the virtual springs during the experiment. During the experiment, the participants were presented with virtual springs with two different stiffness $(2 \mathrm{~N} / \mathrm{mm}$ and $6 \mathrm{~N} / \mathrm{mm})$ with visually same (congruent) and visually different (incongruent). Each condition is presented 4 times in random 


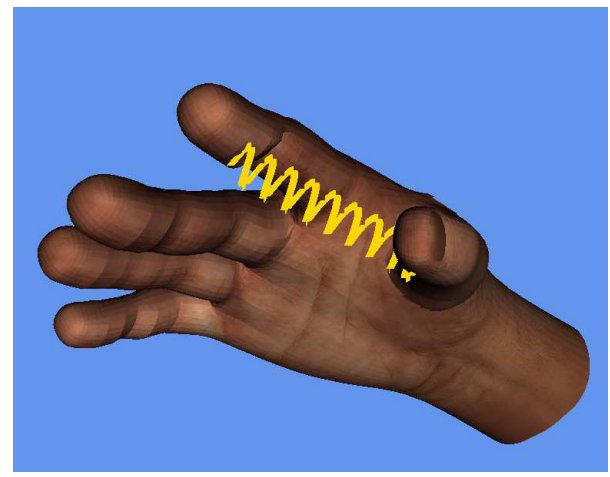

(a) Spring with lower stiffness

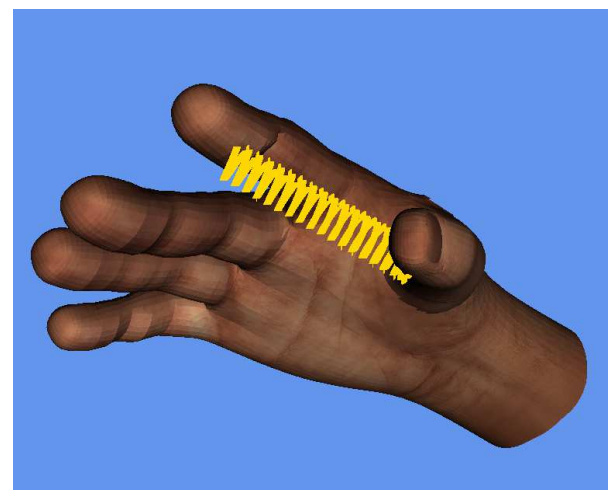

(b) Spring with higher stiffness.

Fig. 4. Virtual springs with different stiffness.

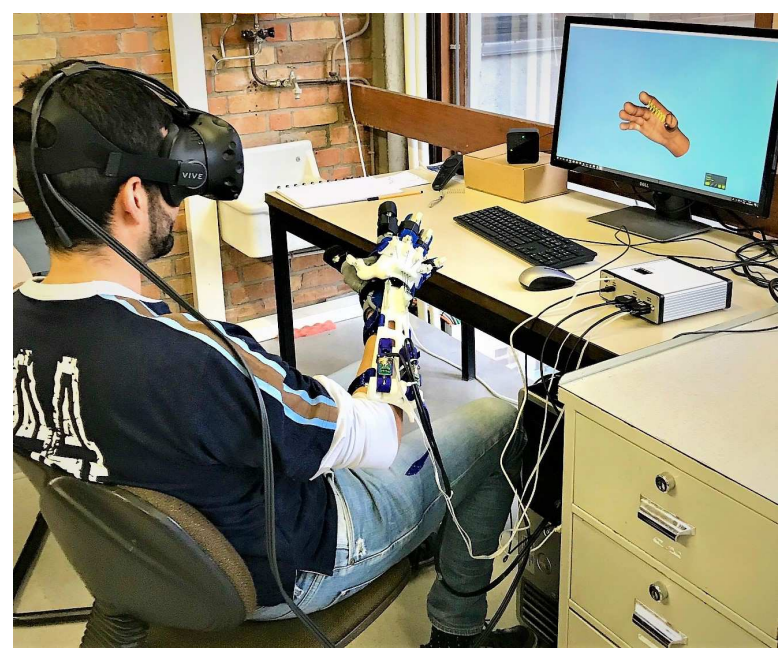

Fig. 5. The experimental setup: In this experiment participants were asked to wear the HTC VIVE headset and ExoTen-Glove and squeeze a set of virtual springs, visually different, with different stiffness by their thumb and index finger.

order. In order to make the compression of the virtual spring same, the movement range of the index finger is constrained from a fully open position to a predefined closed position. Participants were allowed to squeeze it until they are sure about their response. Their response time, applied force and positions were recorded.

\section{E. Analysis and Results}

The result of stiffness experiment is presented in Figure 6. The figure shows the accuracy of the congruent and incongruent conditions. The accuracy is calculated as the percentage of correct answers of matching spring difference with the virtual spring value. White bars represent the conditions in which the stiffness value presented in ExoTen-Glove is visually same (congruent) presented at HTC Vive headset. Grey bars represent the trials in which the stiffness value presented in ExoTen-Glove is visually different (incongruent) presented at head mounted display. Lines on the bars show the standard errors.

Repeated measures two-way analysis of variance (ANOVA) was used for analyzing the mean accuracy and reaction times. The ANOVA design factors were: Congruency (congruent/incongruent), Spring stiffness (low/high). For post-hoc comparisons, paired t-tests were used. Main effect of congruency $(\mathrm{F}(1,7)=12.7, \mathrm{p}<0.01)$ was found with the ANOVA on accuracy. A twoway interaction between Congruency, Spring Stiffness $(F(1,7)=0.163, p=0.69)$ was not found. A main effect of spring stiffness $(\mathrm{F}(1,7)=5.47, \mathrm{p}<0.05)$ was found with the ANOVA performed on the reaction times. We did not get a two-way interaction between Congruency, Spring Stiffness $(\mathrm{F}(1,7)=0.167, \mathrm{p}=0.7)$. We performed paired t-test comparisons between the congruent and incongruent conditions for the accuracy. This analysis revealed that the congruent case differed significantly incongruent case $(\mathrm{t}(7)=5.80, \mathrm{p}<0.001)$. Additionally, paired t-test comparisons between the low and high stiffness conditions for the reaction times revealed that the reaction times for the low stiffness differed significantly from high stiffness case $(\mathrm{t}(7)=5.80, \mathrm{p}<0.001)$.

The Figure 6 shows that the accuracy of the participants detecting spring stiffness for the congruent condition is $80 \%$. The accuracy of detecting spring stiffness decreased to $62.5 \%$ for the incongruent case. Participants had significantly higher accuracy in detecting the spring stiffness difference with the virtual spring when the stiffness is same visually at the HTC VIVE headset. The analysis of reaction times revealed that participants took longer time in detecting the high stiffness compared to low spring stiffness (see Fig. 7).

\section{F. Discussion}

We exploited the ExoTen-Glove for the stiffness value comparison experiment and participants were asked to squeeze two different virtual springs (with different stiffness and visual) with their thumb and index fingers. We expected that the users would easily distinguish stiffness difference when the stiffness simulated at the ExoTen-Glove is visually same (congruent) as the HTC VIVE headset. Our result confirmed our hypothesis. Users had higher accuracy in detecting spring difference when the stiffness value simulated at the ExoTen-Glove was visually same. The result of the present study illustrated that users use not only haptic cues but also visual cues in detecting spring stiffness difference. 


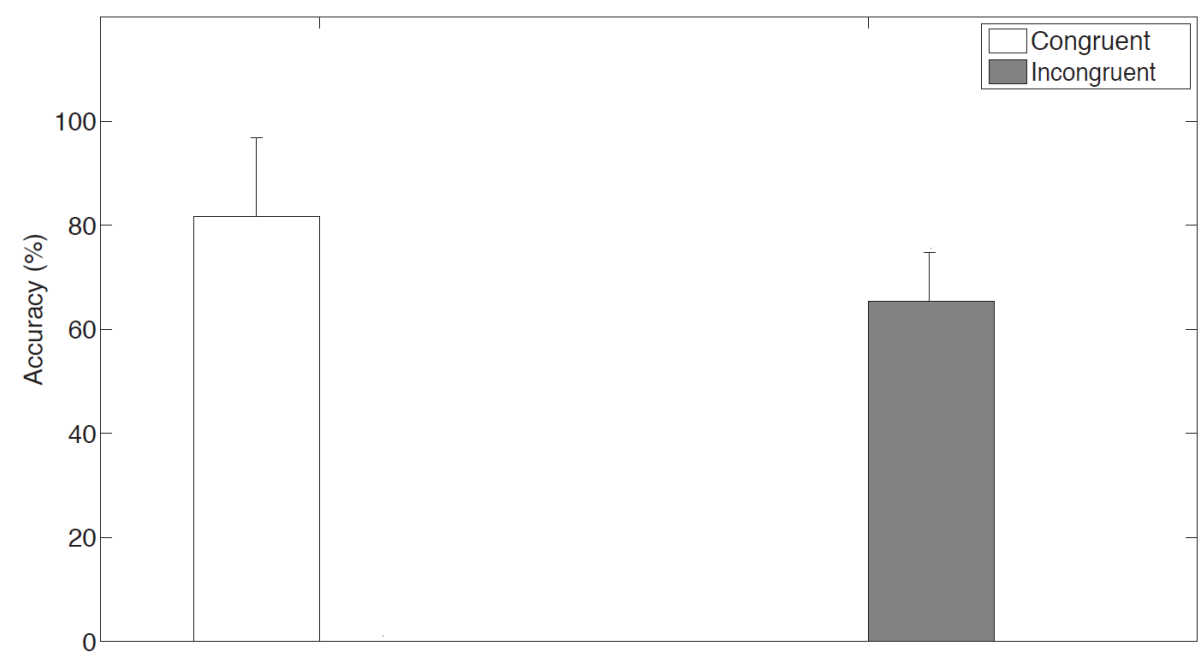

Fig. 6. Percentage of correct responses of stiffness difference estimation.

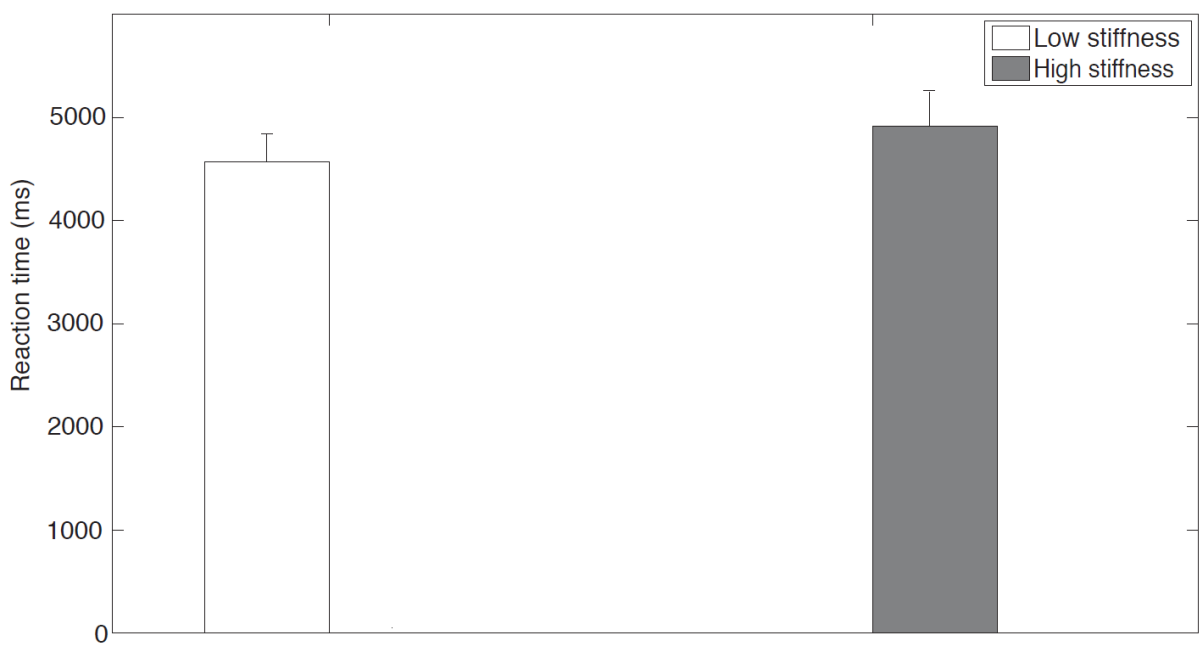

Fig. 7. Response time in milliseconds for low and high stiffness springs.

\section{CONClusions}

It is a challenging task to develop a wearable haptic glove that is lightweight and compact enough to be worn and at the same time powerful enough for enabling stable and high force feedback during grasping virtual objects. We implemented a lightweight and compact wearable haptic glove based on TSA system that is a lightweight and lowcost actuation system, suitable for highly integrated robotic devices like robotic hands and exoskeletons.

Furthermore, understanding human perceptual system is important in order to design more immersive haptic and virtual reality system. Hence in this study we conducted experiments to study the effectiveness of the ExoTen-Glove and the role of visual and haptic cues in stiffness difference discrimination. Virtual spring was compress with the participants thumb and index fingers during the experiment. The aim of the study is to illustrate the benefit of using TSA sys- tem in distinguishing stiffness difference and the role of the visual feedback. The users differentiated stiffness difference better when the haptic feedback from the ExoTen-Glove and visual feedback presented at the HTC VIVE Headset are look-alike. This finding shows that visual and haptic cues play a key role in detecting spring stiffness difference. As next steps in this study, we are optimizing the ExoTen-Glove further by reducing its weight with smaller actuation module and adding composite linkages for increasing realism of haptic feedback. Additionally, we are integrating Unity game engine into CHAI3D platform for complex hand animations with haptic feedback to increase the realism and immersion of the VR. We are also planning to improve the controller performance using a model-based control method.

\section{ACKNOWLEDGEMENT}

The support from VLAIO/Flanders Make Project FINROP ICON is gratefully acknowledged. 


\section{REFERENCES}

[1] H. Kawasaki, "Force feedback glove for manipulation of virtual objects," Jour. Of Robotics and Mechatronics, vol. 5, no. 1, pp. 79-84, 1993.

[2] M. Achibet, G. Casiez, and M. Marchal, "DesktopGlove: a Multifinger Force Feedback Interface Separating Degrees of Freedom Between Hands," in 3DUI'16, the 11th Symposium on 3D User Interfaces, ser. Proc. of the Symposium on 3D User Interfaces, I. C. Society, Ed., Greenville, United States, 2016, p. 10.

[3] M. Fontana, A. Dettori, F. Salsedo, and M. Bergamasco, "Mechanical design of a novel hand exoskeleton for accurate force displaying," in Robotics and Automation, 2009. ICRA'09. IEEE International Conference on. IEEE, 2009, pp. 1704-1709.

[4] P. Galambos, "Vibrotactile feedback for haptics and telemanipulation: Survey, concept and experiment," Acta Polytechnica Hungarica, vol. 9, no. 1, pp. 41-65, 2012.

[5] R. Montao-Murillo, R. Posada-Gmez, A. Martnez-Sibaja, B. GonzalezSanchez, A. Aguilar-Lasserre, and P. Cornelio-Martnez, "Design and assessment of a remote vibrotactile biofeedback system for neuromotor rehabilitation using active markers," Procedia Technology, vol. 7, pp. $96-102,2013$.

[6] P. Olsson, S. Johansson, F. Nysjö, and I. Carlbom, "Rendering stiffness with a prototype haptic glove actuated by an integrated piezoelectric motor," in Proc. Int. Conf. on Haptics: Perception, Devices, Mobility, and Communication, ser. EuroHaptics'12, 2012, pp. 361-372.

[7] C. Tejeiro, C. E. Stepp, M. Malhotra, E. Rombokas, and Y. Matsuoka, "Comparison of remote pressure and vibrotactile feedback for prosthetic hand control," in 2012 4th IEEE RAS EMBS International Conference on Biomedical Robotics and Biomechatronics (BioRob), 2012, pp. 521-525.

[8] G. A. Tabot, J. F. Dammann, J. A. Berg, F. V. Tenore, J. L. Boback, R. J. Vogelstein, and S. J. Bensmaia, "Restoring the sense of touch with a prosthetic hand through a brain interface," Proceedings of the National Academy of Sciences, vol. 110, no. 45, pp. 18279-18 284, 2013.

[9] I. Ivanisevic and V. J. Lumelsky, "Configuration space as a means for augmenting human performance in teleoperation tasks," Trans. Sys. Man Cyber. Part B, vol. 30, no. 3, pp. 471-484, 2000.

[10] R. V. Dubey, S. E. Everett, N. Pernalete, and K. A. Manocha, "Teleoperation assistance through variable velocity mapping," IEEE Trans. on Robotics and Automation, vol. 17, no. 5, pp. 761-766, 2001.

[11] A. Bardorfer, M. Munih, A. Zupan, and A. Primozic, "Upper limb motion analysis using haptic interface," IEEE/ASME Trans. on Mechatronics, vol. 6, no. 3, pp. 253-260, 2001

[12] A. Sengül, M. Hosseini, M. Bouri, Z. Kowalczuk, and H. Bleuler, "Movement perception with the use of a motorized delta armrest and virtual reality," in Human System Interaction (HSI), 2013 The 6th International Conference on. IEEE, 2013, pp. 78-83.

[13] M. Guthold, M. R. Falvo, W. G. Matthews, S. Paulson, S. Washburn, D. A. Erie, R. Superfine, F. P. Brooks, and R. M. Taylor, "Controlled manipulation of molecular samples with the nanomanipulator," IEEE/ASME Trans. on Mechatronics, vol. 5, no. 2, pp. 189-198, 2000.

[14] S. Marlière, D. Urma, J. Florens, and F. Marchi, "Multi-sensorial interaction with a nano-scale phenomenon: the force curve," in Proc. of EuroHaptics, 2004, pp. 246-253.

[15] T. H. Massie, J. K. Salisbury et al., "The phantom haptic interface: A device for probing virtual objects," in Proceedings of the ASME winter annual meeting, symposium on haptic interfaces for virtual environment and teleoperator systems, vol. 55, no. 1. Citeseer, 1994, pp. 295-300.

[16] "Cybergrasp," http://www.cyberglovesystems.com , accessed: 201804-01.

[17] M. Bouzit, G. Burdea, G. Popescu, and R. Boian, "The rutgers master ii-new design force-feedback glove," IEEE/ASME Transactions on mechatronics, vol. 7, no. 2, pp. 256-263, 2002.

[18] I. Choi, E. W. Hawkes, D. L. Christensen, C. J. Ploch, and S. Follmer, "Wolverine: A wearable haptic interface for grasping in virtual reality," in Intelligent Robots and Systems (IROS), 2016 IEEE/RSJ International Conference on. IEEE, 2016, pp. 986-993.

[19] X. Gu, Y. Zhang, W. Sun, Y. Bian, D. Zhou, and P. O. Kristensson, "Dexmo: An inexpensive and lightweight mechanical exoskeleton for motion capture and force feedback in vr," in Proceedings of the 2016 CHI Conference on Human Factors in Computing Systems, ser. CHI '16. New York, NY, USA: ACM, 2016, pp. 1991-1995. [Online]. Available: http://doi.acm.org/10.1145/2858036.2858487
[20] G. Palli, C. Natale, C. May, C. Melchiorri, and T. Würtz, "Modeling and control of the twisted string actuation system," IEEE/ASME Trans. on Mechatronics, vol. 18, no. 2, pp. 664-673, 2013.

[21] S. Moshe, "Twisting wire actuator," Journal of Mechanical Design, vol. 127, no. 3, p. 441445, jul 2004.

[22] G. Palli, C. Melchiorri, G. Vassura, U. Scarcia, L. Moriello, G. Berselli, A. Cavallo, G. De Maria, C. Natale, S. Pirozzi, C. May, F. Ficuciello, and B. Siciliano, "The DEXMART Hand: Mechatronic design and experimental evaluation of synergy-based control for human-like grasping," The Int. Journal of Robotics Research, vol. 33, no. 5, pp. 799-824, 2014.

[23] D. Popov, I. Gaponov, and J. Ryu, "A preliminary study on a twisted strings-based elbow exoskeleton," in World Haptics Conference (WHC), 2013. IEEE, 2013, pp. 479-484.

[24] A. Pepe, M. Hosseini, U. Scarcia, G. Palli, and C. Melchiorri, "Development of an haptic interface based on twisted string actuators," in Advanced Intelligent Mechatronics (AIM), 2017 IEEE International Conference on. IEEE, 2017, pp. 28-33.

[25] M. Hosseini, R. Meattini, G. Palli, and C. Melchiorri, "A wearable robotic device based on twisted string actuation for rehabilitation and assistive applications," Journal of Robotics, vol. 2017, 2017.

[26] _ "Development of semg-driven assistive devices based on twisted string actuation," in Control, Automation and Robotics (ICCAR), 2017 3rd International Conference on. IEEE, 2017, pp. 115-120.

[27] M. Hosseini, G. Palli, and C. Melchiorri, "Design and implementation of a simple and low-cost optoelectronic force sensor for robotic applications," in Advanced Intelligent Mechatronics (AIM), 2016 IEEE International Conference on. IEEE, 2016, pp. 1011-1016.

[28] G. Palli, M. Hosseini, and C. Melchiorri, "A simple and easy-to-build optoelectronics force sensor based on light fork: Design comparison and experimental evaluation," Sensors and Actuators A: Physical, vol. 269, pp. 369-381, 2018. 